\title{
Colesteatoma de oído con complicación intracraneana en contexto de pandemia COVID-19: Reporte de caso clínico y revisión de literatura
}

\section{Ear cholesteatoma with intracranial complication in the context of COVID-19 pandemic: Case report and literature review}

\author{
Diego Navarro A. ${ }^{1}$, Jorge Hidalgo S. ${ }^{1}$, Ilson Sepúlveda A. ${ }^{2,3}$, Thomas Schmidt P., ${ }^{1,3}$
}

'Departamento de Otorrinolaringología, Facultad de Medicina, Universidad de Concepción. Concepción, Chile.

${ }^{2}$ Facultad de Odontología, Universidad Finis Terrae; ${ }^{3}$ Servicio de

Otorrinolaringología y Cirugía de Cabeza y Cuello. Hospital Regional Guillermo Grant Benavente, Universidad de Concepción. Concepción, Chile.

Los autores declaran no tener conflictos de interés.

Recibido el 14 de noviembre de 2020. Aceptado el 11 de enero de 2021

Correspondencia: Thomas Schmidt P. Departamento

Otorrinolaringología,

Facultad de Medicina, Universidad de Concepción. Concepción, Chile. Email: schimdtp@udec.cl

\section{Resumen}

La otitis media crónica colesteatomatosa (OMCC) complicada es una condición inusual en la época actual. Clásicamente las complicaciones se han clasificado en extracraneales e intracraneales. Estas últimas suponen un alto riesgo para los pacientes debido a la alta morbimortalidad y las secuelas neurológicas asociadas. La atención médica oportuna y derivación a otorrinolaringología junto con las medidas preventivas en edad pediátrica han disminuido la incidencia de los colesteatomas y sus complicaciones. Se presenta a continuación el caso de una paciente de 50 años, atendida en el Hospital Guillermo Grant Benavente de Concepción (Chile) por cuadro de OMCC complicada con meningoencefalitis y vasculitis infecciosa sin consulta precoz por contexto de pandemia COVID-19. Se expone metodología diagnóstica y manejo terapéutico.

Palabras clave: Otitis media colesteatomatosa, complicaciones intracraneanas, meningitis otogénica, COVID-19.

\begin{abstract}
Complicated chronic cholesteatomatous media otitis (CCMO) is an unusual condition nowadays. Complications have traditionally been classified as extracranial and intracranial. The last one entails a higher risk for patients due to high morbi-mortality and neurological consequences associated. Suitable medical care and otorhinolaryngological attention among with preventive measures in pediatric age have decreased the incidence of cholesteatomas and their complications. The following, is the case of a 50-year-old patient treated for CCMO at Guillermo Grant Benavente Hospital in Concepcion (Chile) complicated due to meningoencephalitis and infectious vasculitis without early diagnose due to COVID-19 pandemic. Diagnostic methodology and therapeutic management are exposed.
\end{abstract}

Keywords: cholesteatomatous chronic otitis media, intracranial complications, otogenic meningitis, COVID-19.

\section{Introducción}

Las infecciones del oído medio, como otitis media aguda y otitis media crónica supurativa con o sin colesteatoma pueden evolucionar hacia complicaciones intracraneales (CI) con una incidencia reportada entre $0,4 \%$ a $6,4 \%$. Entre las CI, la meningitis es la más común con una prevalencia de $35 \%$ a $46,4 \%^{1,2}$. Las vías de diseminación desde un foco otogénico incluyen invasión local hacia las meninges y parénquima cerebral, diseminación hematógena, tromboflebitis de los vasos sanguíneos y dehiscencia ósea ${ }^{3}$.

El colesteatoma es una lesión no neoplásica compuesta por un epitelio escamoso quera- 
tinizado de ubicación anómala y en algunos casos pueden representar una condición potencialmente peligrosa porque puede erosionar segmentos óseos que se encuentran adyacente al tejido cerebral ${ }^{4-6}$. Se han descrito diversas complicaciones intracraneales tales como meningitis, absceso cerebral, absceso epidural, trombosis del seno venoso lateral, encefalitis y meningoencefalocele. Se ha evidenciado que hasta el 5\% de pacientes con OMCC pueden desarrollar alguna de estas complicaciones intracraneales?.

El tratamiento de la otitis media crónica colesteatomatosa (OMCC) complicada con infecciones intracraneales requiere el uso precoz de antibióticos endovenosos asociado a cirugía que permita la erradicación del foco y eventual drenaje de abscesos cerebrales o trombos sépticos ${ }^{8}$. De este modo, el advenimiento de la antibioticoterapia, el fortalecimiento de los sistemas de salud, los avances radiológicos y el tratamiento oportuno de las otitis medias y disfunción tubáricas en edad pediátrica han llevado a una disminución de la incidencia de OMCC y sus complicaciones asociadas en los países desarrollados ${ }^{9,10}$. La pandemia COVID-19 ha provocado que los pacientes con patologías con potenciales complicaciones consulten tardíamente en los servicios asistenciales por miedo al contagio. Series internacionales han reportado un descenso de las consultas de urgencia en el área de otorrinolaringología (ORL) con cifras que alcanzan hasta un $91 \%$ de reducción ${ }^{11}$.

Se presenta caso de paciente con OMCC, atendido en el Hospital Guillermo Gran Benavente de Concepción, Chile, que en el contexto de la pandemia COVID-19 desarrolló simultáneamente un cuadro de OMC complicada con meningoencefalitis y accidente cerebrovascular por vasculitis infecciosa, el cual fue tratado en forma multidisciplinaria, con la combinación de cirugía y antibioticoterapia.

\section{Caso Clínico}

Paciente femenino de 50 años con antecedentes de otitis media crónica oído derecho (OD), hipertensión arterial y ruralidad. Derivada al servicio de urgencias por cuadro de semanas de evolución caracterizado por oto- rrea purulenta OD, cefalea intensa, disartria, nistagmo fase rápida a izquierda y compromiso del estado general. Paciente no realiza consulta precoz por temor a contagio por SARS-CoV-2. $\mathrm{Al}$ ingreso, paciente con compromiso del estado general, abundante otorrea en OD, asociado a signos de irritación meníngea. Parámetros inflamatorios elevados y punción lumbar sin hallazgos patológicos.

Tomografía computada (TC) de cerebro y hueso temporal evidenció extenso proceso expansivo con densidad de partes blandas a nivel del ático y antro mastoideo derecho, heterogéneo, con compromiso erosivo de la pared posterior del CAE, scutum, tegmen timpani, porciones timpánicas y mastoideas del trayecto intrapetroso del séptimo par craneal y canal semicircular lateral. Compromiso expansivo de la cortical medial sin evidencia de fenómenos trombóticos a nivel del seno lateral y sigmoideo ipsilateral. La resonancia magnética nuclear (RMN) evidenció proceso expansivo hipointenso sin realce en secuencia T1 con saturación grasa y uso de contraste endovenoso; asociado a restricción de la difusión en secuencia DWI (Figura 1).

Se inició tratamiento antibiótico empírico de vancomicina, meropenem y ceftazidima. Paciente evolucionó con compromiso de conciencia, Glasgow 8-9 y hemiplejia braquiocrural izquierda. Se realizó RM de cerebro que evidenció, en secuencia T2 FLAIR, múltiples áreas supratentoriales frontoparietales hiperintensas bilaterales, con predominio derecho y realce meníngeo tras la administración de contraste endovenoso (EV), concordantes con lesiones isquémicas agudas y meningitis (Figura 2).

Tras confirmación diagnóstica, se realizó cirugía radical de oído derecho y se evidenció extenso colesteatoma ático-antro-mastoideo, ausencia de cadena oscicular, erosión canal semicircular lateral y conducto de Falopio en porción horizontal y vertical asociado a abundante contenido purulento que emerge por la ventana oval que se drena y se cubre con fascia (Figura 3).

Cultivo de secreción oído: enterococus avium. Evaluación por infectología indicó cuatro semanas de tratamiento antibiótico con meropenem en dosis de $2 \mathrm{~g}$ cada $8 \mathrm{~h}, \mathrm{y}$ completar 8 semanas con amoxicilina en dosis 
Figura 1. Tomografía axial computada: (A) ventana ósea, lesión osteolítica ático-antro-mastoidea con infiltración de pared posterior del CAE y cápsula ótica (flecha roja). (B) Ventana tejidos blandos contrastada, sin evidencia de infiltración en seno lateral (flecha verde). Resonancia magnética: (C) secuencia T1-FSGD sin evidencia de realce tumoral (flecha amarilla). (D) Secuencia DWI, que demuestra proceso expansivo con restricción de la difusión (flecha blanca).
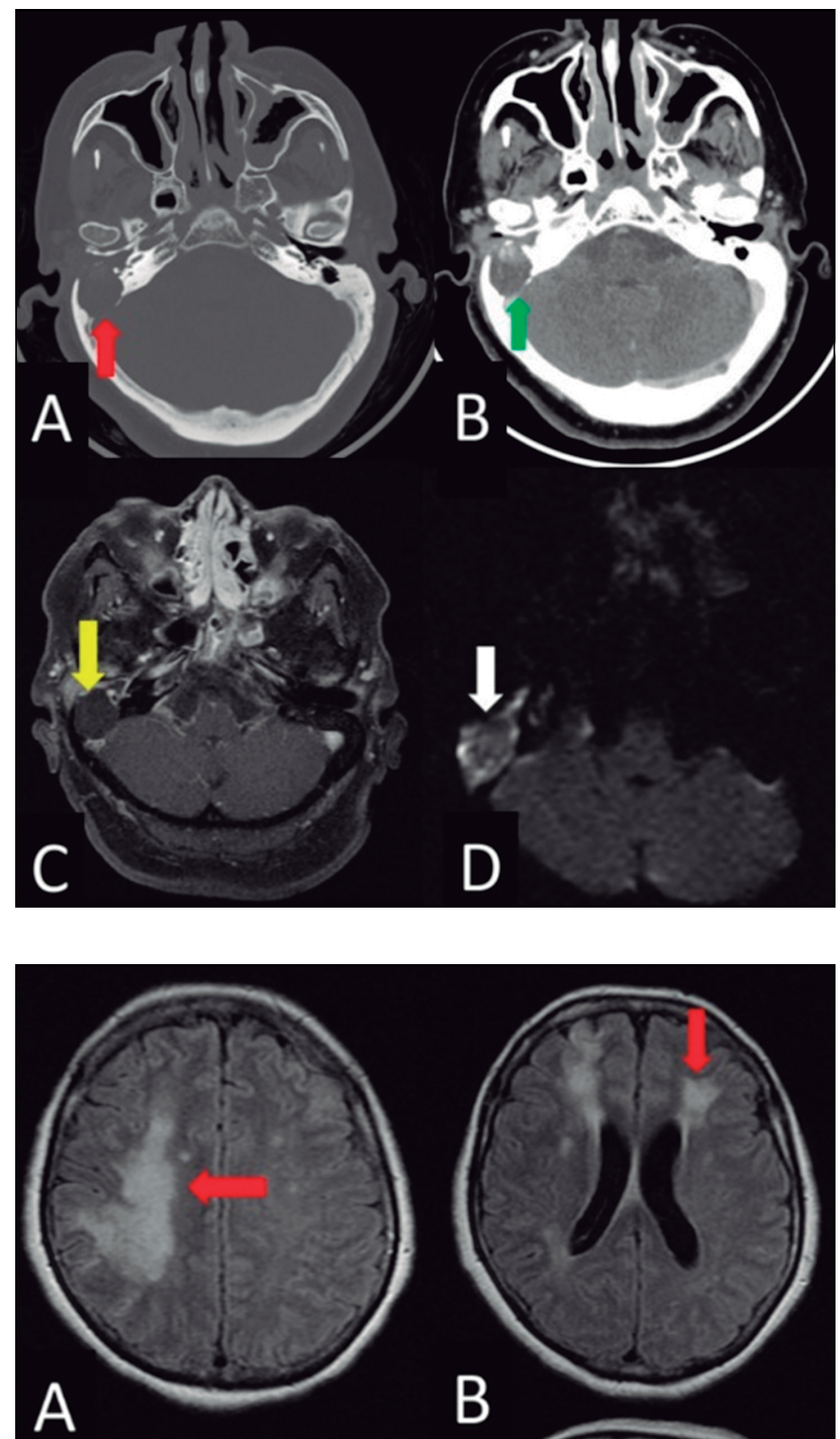

Figura 2. Resonancia magnética. (A) y (B) secuencia T2 FLAIR (Fluid Attenuated Inversion Recovery) múltiples áreas hiperintensas en región frontal izquierda y frontoparietal derecha (flechas rojas). (C) y (D): secuencia T1-GD (gadolinio), realce meníngeo (flechas amarillas). 


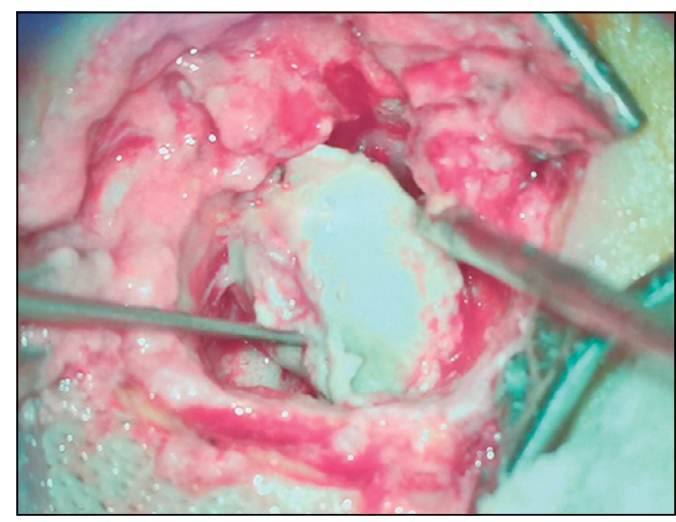

Figura 3. Resección colesteatoma.

de 1 g cada 8 h. Paciente evolucionó afebril, sin compromiso del nervio facial y con parámetros inflamatorios en descenso. Se inició neurorrehabilitación intensiva para manejo de déficit neurológico. Paciente fue evaluada en policlínico seis semanas posteriores al alta con una notoria mejoría del punto de vista neurológico y sin signos de recidiva de colesteatoma en OD.

\section{Discusión}

Otitis media aguda y crónica son enfermedades con potencial riesgo vital por sus complicaciones intracraneales ${ }^{12,13}$. Algunos estudios reportan que la otitis media aguda puede ser responsable del 50\% de las meningitis en adultos y $25 \%$ en niños ${ }^{14}$. El uso extensivo de antibióticos ha disminuido el número de complicaciones derivadas de todas las formas de otitis media ${ }^{15}$. Para el diagnóstico de las CI se requiere un alto índice de sospecha y el uso de imágenes complementarias. TC ofrece una excelente resolución espacial para determinar tamaño, localización de la masa y presencia de erosión ósea, pero es incapaz de entregar información acerca de la naturaleza de la masa ${ }^{6,16,17}$.

En RM las características de la imagen del colesteatoma son inespecíficas y generalmente de carácter iso/hipointenso en secuencia T1 e hiperintenso en secuencia T2 comparado con el parénquima cerebral. Técnicas de imágenes ponderadas en secuencia de Diffusion-Weighted Imaging (DWI), basadas en la secuencia convencional spin-eco (SE) ponderada en T2, se han vuelto populares en la identificación del colesteatoma debido a su mayor precisión y adquisición más corta en relación con la RM contrastada ${ }^{16}$. La introducción de imágenes non-Echo-Planar Imaging (non-EPI-DWI) ha permitido obtener cortes más delgados con mínimos artefactos, logrando optimizar la detección de lesiones pequeñas, con sensibilidad y especificidad mayor al 90\% $\%^{7,16,17,19-21}$. De este modo, esta sería una técnica confiable para el diagnóstico tanto de colesteatoma y la evaluación de sus complicaciones, contribuyendo al otorrinolaringólogo en la toma de decisiones.

Con respecto al tratamiento quirúrgico, la eliminación del proceso supurativo es esencial para la resolución del cuadro infeccioso y cambiar el curso clínico de la enfermedad, incluso si el estudio imagenológico no es concluyente. La pandemia COVID-19 ha repercutido severamente en los distintos sistemas sanitarios, los cuales han debido redistribuir sus recursos y modificar sus modelos de atención para tratar los contagios y disminuir la propagación del virus $^{22,23}$. Se ha reportado una notoria reducción de las atenciones ambulatorias y una disminución de las cirugías electivas ${ }^{11,22,24,26}$. Asimismo, se ha evidenciado un cambio en las conductas de los pacientes que han reducido la asistencia a controles ambulatorios y/o consultas en unidades de urgencia, reportándose hasta un 91\% de disminución de atenciones de urgencias en $\mathrm{ORL}^{11,26}$. Algunas de las causas que explicarían este fenómeno son: miedo al contagio, efectos indeseados de las recomendaciones sanitarias de permanecer en casa, disponibilidad de transporte público, cercanía a centros de salud, entre otros ${ }^{11,25,26}$. Este conjunto de factores se asociaría a cambios epidemiológicos producto de la pandemia COVID-19 que resultaría en un aumento en la incidencia de patologías descompensadas y/o complicadas como el caso presentado.

\section{Conclusión}

Las complicaciones intracraneales derivadas de procesos infecciosos del oído medio son enfermedades amenazantes que tienen una alta tasa de morbimortalidad. La indicación de antibióticos de amplio espectro de manera precoz y la resolución quirúrgica en centros de alta complejidad son fundamen- 
tales. El estudio con técnicas imagenológicas de última generación permite identificar pequeñas lesiones sospechosas de colesteatoma y sus diversas complicaciones. No obstante, no se debe priorizar el análisis radiológico exhaustivo sobre la resolución quirúrgica ante pacientes con alta sospecha clínica de enfermedad complicada.

Los efectos epidemiológicos de la pandemia COVID-19 todavía son inciertos y podrían asociarse a consultas tardías en patologías relevantes en el área de la otorrinolaringología. Es fundamental que en todos los niveles de la red asistencial se genere un ambiente de tranquilidad y seguridad a la población para garantizar atenciones médicas de manera priorizada y oportuna. De acuerdo al caso presentado, se estima que las consultas tardías en contexto de pandemia pueden ser causa de morbimortalidad, secuelas permanentes y estadías prolongadas en unidades críticas. Es necesario realizar estudios prospectivos para obtener resultados concluyentes.

\section{Bibliografía}

1. Bruschini L, Fortunato S, Tascini C, et al. Otogenic Meningitis: A Comparison of Diagnostic Performance of Surgery and Radiology. Open Forum Infect Dis. 2017;4(2):ofx069. doi: 10.1093/ofid/ofx069.

2. Penido $\mathrm{N}$ de $\mathrm{O}$, Borin $\mathrm{A}$, Iha LC, et al. Intracranial complications of otitis media: 15 years of experience in 33 patients. Otolaryngol Head Neck Surg. 2005;132(1):37-42. doi: 10.1016/j.otohns.2004.08.007.

3. Patel KM, Almutairi A, Mafee MF. Acute otomastoiditis and its complications: role of imaging. Operative Techniques in Otolaryngology. 2014;25:21-28.

4. Watanabe K, Hatano GY, Fukada N, Kawasaki T, Aoki H, Yagi T. Brain abscess secondary to the middle ear cholesteatoma: a report of two cases. Auris Nasus Larynx. 2004;31(4):433-437. doi: 10.1016/j. anl.2004.09.010.

5. Stott C, Ortega G, Bravo G, Délano PH. Cirugía del colesteatoma y audición. Rev Otorrinolaringol Cir Cabeza Cuello. 2013;73(3):243-248. doi: 10.4067/ S0718-48162013000300006.

6. Cabrera N, Sedano C, Délano PH, Alvo A. Uso de resonancia magnética con secuencia de difusión no-ecoplanar para la detección de colesteatoma en pacientes con cirugía de oído previa: Presentación de 4 casos. Rev Otorrinolaringol Cir Cabeza Cuello. 2015; 75:145-155. 10.4067/S0718-48162015000200009.

7. Mustafa A, Heta A, Kastrati B, Dreshaj SH.
Complications of chronic otitis media with cholesteatoma during a 10-year period in Kosovo. Eur Arch Otorhinolaryngol. 2008;265:1477-1482.

8. Prasad SC, Shin SH, Russo A, Di Trapani G, Sanna M. Current trends in the management of the complications of chronic otitis me- dia with cholesteatoma. Curr Opin Otolaryngol Head Neck Surg. 2013;21(5):446-454.

9. Kirti YK, Yashveer JK. Dilemmas in Current Management of Complicated Chronic Otitis Media. Indian J Otolaryngol Head Neck Surg. 2019;71(2):155-160. doi: 10.1007/s12070-018-1334-3.

10. Prasad SC, Shin SH, Russo A, Di Trapani G, Sanna M. Current trends in the management of the complications of chronic otitis media with cholesteatoma. Curr Opin Otolaryngol Head Neck Surg. 2013;21(5):446-54. doi: 10.1097/ MOO.0b013e3283646467.

11. Elli F, Turri-Zanoni M, Arosio AD, Karligkiotis A, Battaglia P, Castelnuovo P. Changes in the use of Otorhinolaryngology Emergency Department during the COVID-19 pandemic: report from Lombardy, Italy. Eur Arch Otorhinolaryngol. 2020;277(12):35253528. doi: 10.1007/s00405-020-06119-z.

12. Geyik MF, Kokoglu OF, Hosoglu S, Ayaz C. Acute bacterial meningitis as a complication of otitis media and related mortality factors. Yonsei Med J. 2002;43:573-8.

13. Syal R, Singh H, Duggal KK. Otogenic brain abscess: management by otologist. J Laryngol Otol. 2006;120:837-41.

14. Moya AJ, Curiel JA, Remiro RG, et al. [Trombosis de seno sigmóide como compli- cación de otitis media]. Ann Esp Pediatr. 2000;53:488-91.

15. Osma U, Cureoglu S, Hosoglu S. The complications of chronic otitis media: report of 93 cases. J Laryngol Otol. 2000;114:97-100.

16. Baráth K, Huber AM, Stämpfli P, Varga Z, Kollias S. Neuroradiology of cholesteatomas. AJNR Am J Neuroradiol. 2011;32(2):221-229. doi: 10.3174/ajnr. A2052.

17. Moreno-Ramos MD, Pérez MO, Ibáñez Rodríguez JA, Gómez Galán MJ, Ramos Medrano FJ. Diffusionweighted magnetic resonance imaging with echo-planar and non-echo-planar (PROPELLER) techniques in the clinical evaluation of cholesteatoma. Arch Otolaryngol Rhinol. 2019;5:14-19. doi: 10.17352/2455-1759.000089.

18. Ahualli J. Aspectos generales de las secuencias de difusión de imagen en resonancia magnética. Revista Argentina de Radiología. 2010;74(3):227-237.

19. Touska P, Connor SEJ. Imaging of the temporal bone. Clin Radiol. 2020;75(9):658-674. doi: 10.1016/j. crad.2020.06.013.

20. Sheng Y, Hong R, Sha Y, Zhang Z, Zhou K, Fu C. Performance of TGSE BLADE DWI compared with RESOLVE DWI in the diagnosis of 
cholesteatoma. BMC Med Imaging. 2020;20(1):40. doi: 10.1186/s12880-020-00438-7.

21. Juliano AF. Cross Sectional Imaging of the Ear and Temporal Bone. Head Neck Pathol. 2018;12(3):302320. doi:10.1007/s12105-018-0901-y.

22. Kasle DA, Torabi SJ, Savoca EL, Judson BL, Manes RP. Outpatient Otolaryngology in the Era of COVID-19: A Data-Driven Analysis of Practice Patterns. Otolaryngol Head Neck Surg. 2020;163(1):138-144. doi: $10.1177 / 0194599820928987$.

23. Centers for Disease Control and Prevention. Implementation of mitigation strategies for communities with local COVID-19 transmission. Accessed March 27, 2020. https://www.cdc.gov/ coronavirus/2019-ncov/downloads/communitymitigation-strategy.pdf
24. De Bernardi F, Turri-Zanoni M, Battaglia P, Castelnuovo P. How to Reorganize an Ear, Nose, and Throat Outpatient Service During the COVID-19 Outbreak: Report From Northern Italy. Laryngoscope. 2020;130(11):2544-2545. doi: 10.1002/lary.28716.

25. Lange SJ, Ritchey MD, Goodman AB, et al. Potential Indirect Effects of the COVID-19 Pandemic on Use of Emergency Departments for Acute Life-Threatening Conditions - United States, January-May 2020. MMWR Morb Mortal Wkly Rep. 2020;69(25):795-800. doi: 10.15585/mmwr. $\mathrm{mm} 6925 \mathrm{e} 2$.

26. Czeisler MÉ, Marynak K, Clarke KEN, et al. Delay or Avoidance of Medical Care Because of COVID-19Related Concerns - United States, June 2020. MMWR Morb Mortal Wkly Rep. 2020;69(36):1250-1257. doi: 10.15585/mmwr.mm6936a4. 